\title{
NANOMETROLOGÍA: IMPACTO EN LOS SISTEMAS DE PRODUCCIÓN
}

\author{
NANOMETROLOGY: IMPACT ON PRODUCTION SYSTEMS
}

\author{
Estefanía Ruiz Gómez', Luis Fernando Giraldo Jaramillo²
}

Fecha de recepción: 4 de marzo de 2016

Fecha de revisión: 27 de abril de 2016

Fecha de aprobación: 16 de junio de 2016

Referencia: E. Ruiz Gómez, L. F. Giraldo Jaramillo (2016). Nanometrología: Impacto en los sistemas de producción. Ciencia e Ingeniería Neogranadina, 26 (2), pp. 49-72, DOI: http://dx.doi.org/10.18359/rcin.1771

\section{RESUMEN}

El presente trabajo es el resultado de una revisión bibliográfica sobre: la aplicación de la nanometrología en diferentes sectores industriales y cómo esta contribuye a alcanzar los estándares de calidad de los productos a través de la medición de parámetros críticos en los procesos productivos; así como una descripción de los retos a los que se enfrenta esta ciencia en diferentes sectores. Se reportan los avances y el desarrollo de nuevas técnicas que permiten medir las características de los nanodispositivos, nanomateriales y equipos, lo que ha permitido potenciar el desarrollo de industrias, una disminución de los costos y la automatización de los procesos. Dentro del texto se hace referencia a industrias en progreso y el efecto que tiene el control de parámetros para la optimización de los procesos, el diseño de nanodispositivos y la creciente necesidad de la reglamentación en el uso de nanocompuestos, el diseño de técnicas confiables y de protocolos para el uso de nanopartículas. El adelanto de la nanometrología ha contribuido al desarrollo de materiales de referencia, herramientas que aumenten la exactitud y precisión de las medidas, así como técnicas para la calibración de las herramientas y equipos apropiados para las medidas a nanoescala, lo que se traduce en un proceso productivo controlado

1. Ing. de Producción, Semillero de Investigación: Gestión Metrológica, Instituto Tecnológico Metropolitano, Medellín, Colombia, estefaniaruiz98510@ correo.itm.edu.co

2. Ing. Electrónico, M.Sc., Tutor Semillero de Investigación: Gestión Metrológica, Instituto Tecnológico Metropolitano, Medellín, Colombia, luisgiraldo@itm.edu.co 
que garantiza la calidad de los productos. Finalmente, se incluye el estado de la nanometrología en Colombia, enfocada en procesos industriales, como: el sector de alimentos, textil, salud y producción de nanomateriales, así como el trabajo desarrollado por grupos de investigación.

Palabras clave: nanometrología, nanotecnología, nanofabricación, medición.

\section{ABSTRACT}

This work is the result of a review of the literature on the application of nanometrology in different industrial sectors and how this one contributes to reach the standards of quality of the products through the measurement of critical parameters in the productive processes, as well as a description of the challenges this science faces in different sectors. The progress and development of new techniques that allow the measurement of the characteristics of nanodevices, nanomaterials, and equipment are reported, ones that have allowed to promote the development of industries, a decrease of costs, and the automation of processes. Within the text, there is reference to industries in progress and the effect that the control of parameters exerts for the optimization of the process, the design of a nanodevice, the growing need for regulation in the use of nanocomposites, and the designing of reliable technical and protocols for the use of nanoparticles. The advancement in nanometrology has contributed to the development of reference materials, tools that increase the accuracy and precision of the measurements, as well as techniques for the calibration of tools and equipment suitable for measurements at nanoscale, which translates into a controlled production process that ensures the quality of the products. Finally, the state of nanometrology in Colombia is included, focusing on industrial processes such as the sector of food, textile, health, and production of nanomaterials, as well as the work developed by research groups.

Keywords: nanometrology, nanotechnology, nanomanufacturing, measurement.

\section{INTRODUCCIÓN}

La nanotecnología ha despertado gran interés en diversos campos industriales, como la electrónica, el sector de alimentos, la industria cosmética, la medicina, entre otros. Los desarrollos tecnológicos en estos campos exigen que se realicen mediciones con un enfoque holístico, en el cual se asegure metrológicamente la cadena de suministro a nanoescala, en busca de aumentar la confiabilidad de las mediciones, siendo este el punto de partida para el ajustado control sobre los procesos de transformación industrial. Lo anterior en cumpliendo de exigentes estándares de calidad que permiten a las empresas productoras del país ingresar a nuevos mercados a nivel internacional, conforme lineamientos establecidos por el Subsistema Nacional de la Calidad (SNCA) en Colombia. Por otra parte, el enfoque científico de la metrología permite que se mejore el sistema de medición utilizado en cualquier campo del saber, orientando las actividades 
investigativas al desarrollo de nuevas técnicas y procedimientos de medición, así como al establecimiento de patrones de medida y materiales de referencia que permitan generar trazabilidad en la medición, y con esto asegurar un porcentaje específico en la confiabilidad de las mediciones.

Teniendo en cuenta el auge de la nanotecnología y su impacto económico, tecnológico y social en el sector industrial y académico, se hace necesario asegurar el resultado de mediciones realizadas a magnitudes físicoquímicas influyentes en la calidad de un producto a nanoescala. Por tal motivo, se considera necesario indagar sobre el aporte de la metrología desde el ámbito de la industria y la ciencia, lo cual constituye al presente documento como un artículo de revisión a nivel internacional, en el cual se exponen y referencian resultados de investigación que pueden ser el punto de partida para desarrollos y/o aplicaciones enfocadas a contribuir con el mejoramiento de la ciencia de la medición a nanoescala y los cuales relacionan el uso de equipos de medición, la apropiación de técnicas y avances en el área de la nanometrología; además, informa sobre los retos a los que se ve enfrentada como ciencia de la medición y los vacíos que requieren de investigación según las actuales necesidades de la industria.

El artículo comprende la siguiente estructura: inicialmente se da una aproximación a la importancia de la nanometrología en los sistemas productivos, así como el alcance en la aplicación de técnicas de medición, el uso de equipos de medida, patrones y materiales de referencia, elementos influyentes en la confiabilidad de las mediciones. Dentro de este apartado se describe la contribución a diversos sectores industriales a través del uso de herramientas y la implementación de sistemas de detección virtual para apoyar los procesos de medición. Se describe la contribución de la nanometrología en la electrónica, siendo ésta la industria que reporta los principales progresos; además, se exponen las herramientas en las que se apoya la nanometrología para realizar procedimientos de medición.

En el segundo apartado se expone el papel de la nanometrología en áreas de constante crecimiento, como la medicina y el sector de alimentos, y se detalla información sobre las técnicas que se emplean, las necesidades en el desarrollo de protocolos de medición y validación de resultados. El presente documento también relaciona los estándares existentes a nivel internacional, para la construcción de nanomateriales de referencia, los cuales sirven como base para el diseño de procedimientos de calibración o comparaciones a nanoescala. En el apartado final se describe el estado de la nanometrología en Colombia, los organismos que investigan y el aporte de la academia en este campo. Por último se presentan las conclusiones y las referencias que componen esta investigación.

\section{MATERIALES Y MÉTODOS}

\subsection{Metodología}

Se llevó a cabo una búsqueda en las bases de datos Scopus, Science Direct, EBSCO, DOAJ, IEEE y Engineering Village, con publicaciones que incluyeran las palabras: nanometrología, nanotecnología, nanofabricación y una combinación de las mismas. De los textos resultantes se seleccionaron los que tuviesen aplicaciones industriales, se incluyeron artículos de revisión, investigaciones teóricas y experimentales, conferencias y reportes de seminarios, y a partir de estos se indagó en las 
técnicas que se aplicaban para la fabricación de nanodispositivos en el periodo comprendido entre 2005 y 2016. Se incluyen algunos documentos examinados en el periodo del 2000 al 2004, los cuales hacen referencia al inicio de la nanometrología en el sector de nanotecnología. No se abarcó toda la temática relacionada con la microscopía, pues otros autores ya han presentado investigaciones sobre este tema. Posterior a ello se complementó la búsqueda con publicaciones referentes al aseguramiento metrológico en procesos productivos, calibración y trazabilidad de las medidas. Finalmente, se analizaron y reportaron en este documento.

\section{RESULTADOS Y DISCUSIÓN}

\subsection{Importancia de la nanometrología en los sistemas productivos}

La nanometrología como ciencia es aplicada a múltiples disciplinas a menor escala, algunas de estas son: la óptica, la electrónica, la salud, la biología, las ingenierías, la química, etc. [1]. Lo anterior permite evidenciar un amplio panorama sobre el cual se pueden desarrollar actividades a nivel investigativo y de desarrollo tecnológico, como respuesta a las necesidades tanto del sector productivo como de la sociedad. La ciencia de la medición a nanoescala (nanometrología) apoya investigaciones desarrolladas por la industria y la academia, e integra técnicas, métodos, materiales de referencia, equipos de medición, entre otros. La sinergia entre los anteriores elementos que conforman el sistema de medida a nanoescala, busca aumentar la confiabilidad en los resultados que caracterizan magnitudes físico-químicas (Figura 1); así, pues, el potencial de esta ciencia se refleja en el desarrollo de nanomateriales, el avance en herramientas para la medición y calibración de productos a nanoescala, la elaboración de estándares de referencia [2] y el uso de patrones para soportar la trazabilidad en las mediciones [3].

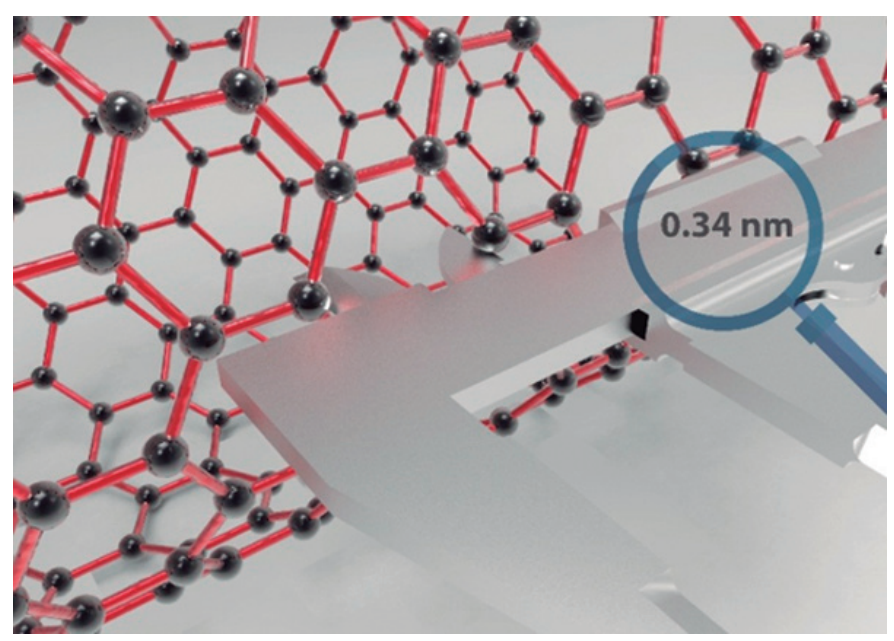

Figura 1. Medición de una partícula a escala nanométrica.

Fuente: Javier Castrillón. Docente ocasional ITM (Facultad de Ciencias Económicas y Administrativas. Figura autorizada para el presente uso). 
Logothetidis menciona que la fabricación de productos y dispositivos a nanoescala depende de la capacidad de medir con precisión y exactitud las propiedades de los mismos [4]. Por otro lado, Ukraintsev y Banke hacen referencia a la calidad de los productos y a la estrecha relación entre las mediciones críticas a nanoescala y la precisión de las herramientas de medición con las cuales se realizan dichas mediciones [5]. Según los criterios expuestos, la nanometrología es la base para el desarrollo de la nanotecnología en los procesos industriales, lo cual implica la necesidad de evolucionar de forma paralela [4].

Siendo uno de los conceptos más desarrollados en la evolución de la calidad, la metrología ha sido el elemento determinante entre calidad de los productos y la mejora de las operaciones dentro de los procesos de transformación. Lo anterior relaciona que el principal concepto a optimizar son los sistemas de medición, en busca de mejorar las cadenas productivas a nanoescala. Estas mejoras hacen referencia al aumento de velocidad en las operaciones dentro de los procesos, aumento en los niveles de precisión y exactitud en la información que entregan los equipos de medición, disminuyendo la incertidumbre y generando confiabilidad de los datos numéricos que relacionan una característica de producto [6].

Actualmente la nanometrología es concebida como una herramienta de apoyo para satisfacer necesidades en el campo de la electrónica, en especial en la construcción de microprocesadores, en los cuales se deben controlar magnitudes, como la longitud y espesor. Este tipo de avances en la electrónica permite mejorar características en equipos y técnicas de medición, como: exploración por sonda (STM, SPM, AFM, KPM, LFM, CMM), haz de iones (AES, IBA, SIMS), haz electrónico
(TEM, HRTEM, SEM, EELS, AES), métodos ópticos (NSOM, Raman, DLS), rayos X (XPS, SRD, XPS, EDX), técnicas electromagnéticas (SET, ELM, MFM, SCM, SKPM, C-AFM), entre otros; además de ser útil para: mejorar el poder de resolución de los microscopios, caracterizar nanomateriales, determinar de forma cuantitativa propiedades mecánicas en algunos materiales, en la deformación plástica de los sólidos, la interacción entre superficies, entre otras [3-4, 7-10].

El desarrollo en este campo resulta importante, porque permitesostenerla actividadeconómica en diferentes sectores, ya que asegura que los resultados son comparables, lo cual posibilita la comercialización de productos, además le permite a las entidades gubernamentales la regulación y normalización de los bienes y servicios. Por otro lado, la investigación en este campo proporciona un respaldo a los referentes normativos y legales de cada país, lo que genera avance permanente al comparar dichos estándares frente a organismos internacionales (Figura 2) [10].

Entre los retos que enfrenta la nanometrología se encuentran: el desarrollo de pruebas para la fabricación de materiales de referencia, diseño de métodos de calibración para medidores a nanoescala, determinación de técnicas de medición reproducibles para cada propiedad del producto o material, según se menciona en el reporte del segundo seminario internacional en metrología llevado a cabo en 2008 [7].

Barsic et al. mencionan que los estándares de referencia en este campo están destinados a instrumentos individuales, los cuales no pueden extrapolarse a otro tipo de instrumentos; además, estos investigadores reiteran la necesidad de investigar sobre la mejora de estándares internacionales, así 


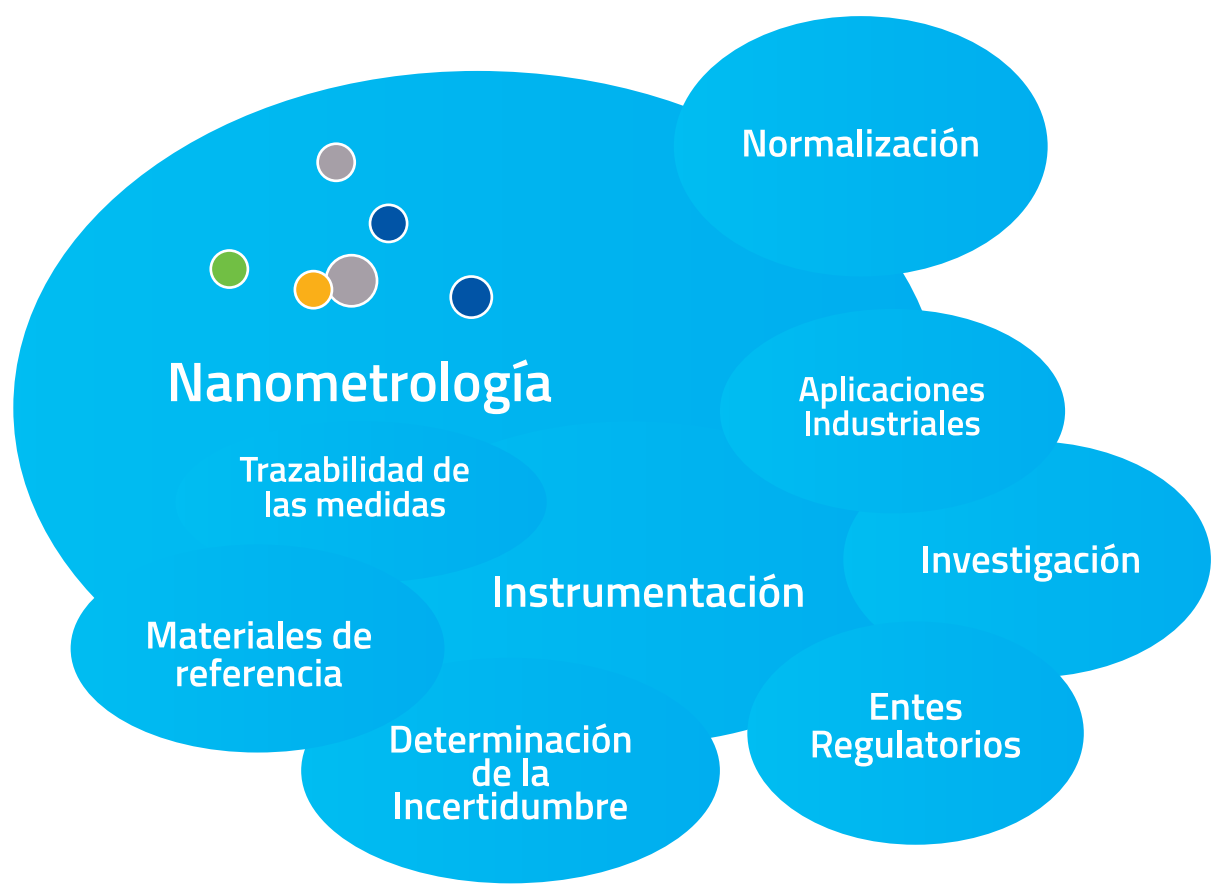

Figura 2. Interacción entre los elementos relacionados con actividades metrológicas.

Fuente: Elaboración propia.

como la generación de garantías sobre la trazabilidad en la unidad de medida a nivel internacional [11]. Varios autores reportan la ausencia de normalización en cuanto a las prácticas ambientales, el descarte y degradación de nanomateriales empleados en el área de la salud y la importancia de definir los patrones de referencia [12-13].

\subsection{Aporte a los sectores industriales}

La ciencia y la tecnología avanzan en la fabricación de nanodispositivos que pueden ser empleados en una gran variedad de industrias; para la fabricación de estos son requeridas máquinas y componentes de tamaño preciso [14], lo que permite la fabricación de nanomateriales, los cuales deben ser medidos, caracterizados y sometidos a diversos procesos de transformación. Un factor común es la disminución en el tamaño de los componentes de la instrumentación y la maquinaria necesaria para transformar, causando como efecto "la disminución del consumo energético y la generación de impactos ambientales positivos" [15].

Hoy día, la ingeniería de los materiales requiere de una caracterización metrológica exacta, a través de la cual se permitan identificar propiedades como: tipología de superficies, desgaste de material (abrasivo, adhesivo, por fricción, por fatiga o por una combinación de los desgastes mencionados) [16]. Por otro lado, para apoyar la nanometrología se han desarrollado herramientas que permiten medir la distribución de los tamaños de los materiales, el monitoreo de las piezas durante el proceso de fabricación y las dimensiones geométricas de los elementos a trabajar, a través de instrumentos como: 
el láser, sistemas ópticos, espectrometría y microscopía, siendo esta instrumentación el punto de partida y el referente para garantizar la calidad de los productos y control durante el proceso de elaboración de los nanomateriales y nanodispositivos, asegurando que los elementos siguen un mismo patrón y se fabrican siguiendo las tolerancias establecidas [15], así como el correcto funcionamiento en el producto final [17]. De manera que la integración de estas herramientas contribuye a aumentar los niveles de precisión en la fabricación en línea y a una reducción de la incertidumbre, así como una disminución en el tiempo del proceso y aumentar la eficiencia de la fabricación de nanodispositivos, al identificar errores en los productos antes de ser llevados al mercado [18].

\subsubsection{Industrias potenciales}

Uno de los sectores productivos donde se evidencia la contribución de la nanometrología es en la manufactura de semiconductores, los cualessonempleadosenloscircuitosintegrados (CI), sensores microelectromecánicos (MEMS), nanochips, telecomunicaciones, electrónica automotriz, plantas de energía solar, productos para la iluminación, entre otros, observando en su cadena productiva la implementación de actividades de aseguramiento metrológico [19]. En este campo de la electrónica a nanoescala, la nanometrología se enfrenta a grandes retos, como:

- Impacto de las magnitudes físicas sobre las mediciones electrónicas.

- Identificación de patrones que permiten orientar el rendimiento de los chips.

- Mejora en la confiabilidad de mediciones realizadas a través de instrumentos virtuales.
La fabricación de productos a nanoescala debe cumplir con requisitos como: asegurar la cantidad de componentes establecidos para el producto y su confiabilidad a través de procesos de producción repetibles y reproducibles. Lo anterior se logra a través de la implementación de estándares tanto para el sistema de medición como para el control del proceso productivo, lo que permite que se satisfagan los requisitos establecidos por las partes interesadas. Por tanto, la instrumentación, la ciencia de medición y las normas técnicas conforman la estructura para la nanofabricación.

Entre las aplicaciones que tiene la metrología a nanoescala, se encuentra que es empleada para medir el espesor y la adhesión de una película de material, el tamaño del poro, las propiedades elásticas y medición de los coeficientes de expansión térmica para los nuevos materiales con los que se fabrican los nanochips. Las mediciones de estas propiedades son realizadas por herramientas como el microscopio electrónico de barrido (SEM), el microscopio de fuerza atómica (AFM), el microscopio de efecto túnel (STM) y el microscopio electrónico de transmisión (TEM), que permite conocer el funcionamiento y las propiedades a gran escala de los materiales con los que son fabricados estos dispositivos [20]. Adicional a las técnicas de microscopía, también se emplea la nanometrología dimensional, que permite inspeccionar la geometría y el tamaño de las estructuras, lo mismo que la automatización del proceso de inspección, la cual es apoyada por los sistemas asistidos por computador (CAD) y la calidad asistida por computador (CAQ), así como también por los sensores de alta precisión [21]. Por otro lado, la metrología química tiene un gran impacto en este campo, ya que permite conocer de manera precisa la cantidad de sustancias y propiedades 
de los nanomateriales para la producción de pantallas electrónicas (pantallas de cristal líquido y pantallas plasma), el análisis químico de un semiconductor y la cantidad precisa de gas en nano litros dentro de un MEMS, haciendo posible la difusión de materiales de referencia certificados, que cumplan con los estándares ambientales [22]. Carreteiro y colaboradores reportan la espectroscopia como una técnica que da apoyo a la nanometrología química para: "determinar cuantitativamente la concentración de sustancias químicas presentes en las superficies de los materiales" [12]. Pimpin y Srituravanich reportan el uso de dispositivos nano para la medición de "especies gaseosas en el ambiente" a través de sensores [23].

La medición de la calidad en la fabricación de cada oblea en los semiconductores puede ahorrar una gran cantidad de recursos, aumentar la calidad del producto, reducir el costo de producción y disminuir el tiempo de ciclo; por lo que el ajuste de las herramientas para controlar las dimensiones críticas es fundamental para garantizar el rendimiento del producto final [24]. Al integrar este tipo de tecnología en las cadenas productivas se busca detectar artículos por fuera de las especificaciones, que permitan identificar de forma rápida y correcta las causas de los fallos y ayuden a reducir productos inconformes y la repetición del trabajo, manteniendo el proceso en movimiento, lo que equivale a una reducción en costos y un aumento en el rendimiento de la línea productiva [18].

\subsection{Herramientas para el aseguramiento metrológico}

Entre las herramientas utilizadas para evaluar las especificaciones de calidad se encuentra la elipsometría (metrología óptica), la cual utiliza luz polarizada para caracterizar el espesor de películas delgadas y sus propiedades, además monitorea dimensiones críticas, así como medidas precisas de la difracción de la nanoestructura, lo cual permite construir un perfil del objeto medido y caracterizar nanomateriales a través de la electrónica moderna [23-24].

Un nuevo concepto surge con el objeto de mejorar la precisión y velocidad en la producción de semiconductores, su nombre es metrología virtual. Esta es una técnica de medición en la cual se utilizan datos históricos a manera de identificar patrones de medida, los cuales de forma repetitiva describen el sistema a controlar según tendencias y correlaciones, así como información de la maquinaria, para ajustar la posición de las herramientas de fabricación con el fin de identificar posibles fallos y realizar mantenimiento preventivo. Con base en esto es posible observar que la metrología virtual contribuye a la generación de patrones o referentes de medición, los cuales proporcionan, en tiempo real, la información necesaria para que los ingenieros ajusten y controlen sus procesos [19-26]. Esta técnica pretende solucionar la falencia de la metrología en este campo, ya que actualmente los procesos metrológicos aplicados en esta industria aumentan el ciclo del proceso y no le aportan ningún valor agregado a la oblea, además pretende garantizar la calidad de cada oblea dado que en el momento solo se garantiza la calidad de una muestra por lote [24].

Varios autores coinciden en que las técnicas de medición ópticas presentan un menor costo y un aumento en la calidad del producto frente a las técnicas de microscopía, las cuales son costosas, consumen mucho tiempo y 
no permiten la automatización del proceso [25-27]. Otras de las ventajas de las técnicas mencionadas consisten en la optimización de los recursos del proceso, lo que permite desplegar acciones de mantenimiento predictivo basado en mediciones en tiempo real, y con esto detectar variaciones en un proceso particular de manera oportuna [19].

La ciencia de la medición a nanoescala es un factor activo en procesos de litografía, la cual es una técnica empleada para grabar características sobre la superficie de nano piezas como semiconductores, también es utilizada para la fabricación de estructuras que permiten el funcionamiento de los $\mathrm{Cl}$, MEMS y chips [28-31]. Malshe y colaboradores reportan el uso de esta herramienta para moldear materiales blandos, sin necesidad de reprocesar el material [30]. Pimpin y Srituravanich describen la aplicación de esta herramienta en los campos de la electrónica, la medicina, la biotecnología, la óptica, la fotónica, en acelerómetros integrados en automóviles, dispositivos de microespejos digitales empleados en los televisores y proyectores, sensores de presión (usados para medir la presión de los vasos sanguíneos y de los neumáticos), en la fabricación de nanoprototipos, entre otros. Se entiende que cada uno de los anteriores son sistemas nanoproductivos que requieren mediciones, como el ancho de banda y el diámetro de punto particular, siendo los parámetros que se ven afectados por las condiciones ambientales [23]. Con base en lo anterior se hace posible establecer como patrón al comportamiento de un material frente a la variación de condiciones específicas, de esta manera se generan materiales de referencia para realizar comparaciones de los productos fabricados y controlar las variables críticas de los procesos en busca de estandarizarlos.
Un avance significativo es la aplicación de técnicas para medir las propiedades mecánicas a escalananométrica, como la nanoindentación, la cual es implementada en ciencias biológicas, de los materiales, entre otras [32] . Esta técnica tiene una alta "reproducibilidad, que permite el análisis de muestras pequeñas, delgadas y heterogéneas" y de la superficie de las mismas [33]; Xia y colaboradores hacen referencia a la utilidad de este ensayo para la medición de las propiedades mecánicas en menor escala, como: la dureza y rugosidad de superficie del material, siendo esta última una propiedad que afecta directamente la dureza, dado que las mediciones dependerán del primer punto de contacto entre las superficies, por lo que se requieren herramientas que proporcionen la mayor exactitud posible [34]. Lucca et al. reportan el uso de esta prueba para medir las "propiedades de tracción de pequeñas partículas, recubrimientos o superficies que han sido tratadas química, física o térmicamente" [33]. Li y su equipo de trabajo reportan un sistema de medición de profundidad y de calibración para las herramientas empleadas en este campo, a través de estándares de interferómetros ópticos, paso de altura y una técnica de modulación de fuerza para mejorar la precisión, calibrar y reducir la incertidumbre [35]. Lo cual posibilita la trazabilidad de las medidas y la reducción de las incertidumbres, ya que se corrigen, mitigan y controlan los errores asociados a las medidas, de manera que la máquina herramienta realice un mecanizado dentro de los parámetros establecidos [18].

\section{4 Áreas en desarrollo}

Otro campo donde la nanometrología ha logrado despertar un gran interés es en la fabricación de nanotubos, los cuales han sido empleados en la biología para penetrar las 
paredes de la célula, en aplicaciones médicas, en los marcos de las aeronaves, sensores ambientales, transmisores eléctricos [3637], la electrónica y la óptica, ya que se ha comprobado la fuerza de estos materiales así como las propiedades eléctricas y la eficiencia como conductores de calor [14]. Garner y Vogel mencionan que estos nanotubos de carbono son elementos considerados para el uso de nanodispositivos, pero que existen parámetros para reforzar en este campo, como: la definición de las propiedades electrónicas (diámetro, estructura y composición), la distribución en los componentes donde han de ser ensamblados y el ajuste de estos dispositivos con los demás elementos que componen el mecanismo para que estos puedan ser fabricados en línea. En su artículo mencionan que cuando los nanotubos son fabricados en grandes lotes, se requieren técnicas como la espectroscopia óptica o de emisión para identificar las propiedades de cada elemento, pero estas técnicas no abarcan todas las medidas críticas; se requieren instrumentos que permitan definir el contorno de estos nanodispositivos, lo que actualmente se logra con la aplicación de varias técnicas de microscopía como: TEM, AFM y STM [8]. Las cuales medirán las características de calidad para dar paso a la siguiente etapa de producción de estos nanomateriales.

Qiu y sus colaboradores evaluaron la aplicación de nanotubos como sensores para mejorar la técnica de espectroscopía de Raman debido a sus propiedades mecánicas y espectrales [36]. Otros investigadores están estudiando los nanotubos para la fabricación de bombillas de luz, ya que presentan una alta eficiencia energética [38]. Dai et al. divulgan la normalización que han empleado para la fabricación de películas de nanotubos de carbono alineados verticalmente en un reactor, así como el control de los parámetros para la producción en grandes lotes de estos nanomateriales; en su investigación se apoyan en TEM y SEM, para el cálculo de la longitud, el diámetro y el contenido de carbono amorfo para el diseño de los nanotubos de carbono, aseguran que el propósito de su investigación es estandarizar y optimizar el proceso de manufacturación, "lo cual se logra comparando la calidad de las películas (..), evaluando cuatro características diferentes: morfología, pureza, grosor y la uniformidad". Los autores resaltan que las técnicas de microscopía son destructivas, por lo cual usan las espectroscopía de Raman para medir la uniformidad de las celdas y su grado de pureza, junto con el análisis termogravimétrico (TGA) y para cuantificar el espesor de película emplean la perfilometría; en su investigación afirman que las técnicas de microscopía no son tan rápidas y flexibles como estas últimas. Por otro lado, desarrollan la metrología química para la fabricación de un catalizador para la producción de películas de nanotubos, donde hacen alusión a la importancia de "la metrología cuantitativa para la optimización del proceso" [37]. Venkata y colaboradores reportan el uso de nanotubos como sensores de fluorescencia para la detección de patógenos en el tratamiento de aguas, dada sus excelentes propiedades de adsorción [39]. Li y su equipo de trabajo reportan la fabricación de nanotubos a través de la ablación con láser, donde el diámetro es controlado por el tamaño de partícula, y la longitud de la fibra es controlada por el tiempo de deposición del vapor de carbono [40], lo que evidencian la gran aplicación de estos nanomateriales en diversas industrias.

Existen otras áreas de la nanometrología que abarcan el campo de la salud, como la odontología, en la cual se desarrollan implantes dentales en la escala nanométrica, lo que contribuye a la comodidad de los pacientes y 
a un mayor ajuste de los implantes dentales; para esto se han desarrollado técnicas que permiten evaluar las superficies dentales y realizar las mediciones correspondientes por medio de microscopios digitales 3D, sensores ópticos, sistemas de escaneo, sistemas CAD y CAM, de manera que se obtienen imágenes de alta precisión, así como una evaluación de las superficies de los dientes lo que permite determinar la compatibilidad del implante dental [41]. Coelho y colegas mencionan que estos procesos de osteointegración a escala nanométrica desempeñan un papel fundamental en el desarrollo del diseño, ya que es posible identificar las "respuestas biológicas como: el crecimiento, la interacción entre células y la presencia de proteínas, (...) así como para medir las condiciones de consolidación ósea" para conocer la superficie y la rugosidad idónea donde será instalado el implante. Esto sugiere que la nanometrología desempeña un papel fundamental, tanto para la determinación de las características topográficas como las propiedades físico-químicas de los huesos en la determinación del diseño del implante y en el proceso de osteointegración, siendo todas estas características importantes para considerar al fabricar los implantes, de manera que se consuma menos tiempo, material y que el proceso sea más eficiente [42].

En el marco de la medicina se propone el uso de nanopartículas como puntos cuánticos, dada su capacidad de emisión de luz, lo cual puede aprovecharse para la producción de imágenes, posibilitar la ubicación de tumores y una mayor precisión en la extirpación, así como también la unión de nanopartículas a los tumores; para esto la nanomedicina emplea nanosensores para detectar la presencia de sustancias químicas, detección de sustancias resultantes de una enfermedad, reconocimiento de partes del cuerpo afectadas debido a los cambios de volumen, presión, concentración, temperatura, entre otras propiedades. Estas nanopartículas han de ser fabricadas en un proceso controlado, estandarizado y debe reportar medidas bajo niveles aceptables para el uso de estos nanocompuestosen el organismo. Actualmente se reporta un faltante en la reglamentación del impacto en el medio ambiente y la toxicidad de los nanocompuestos que son empleados para diversas actividades médicas, como la detección de enfermedades, administración de fármacos, agentes de contraste [43], aunque la literatura reporta estudios sobre la toxicidad de diversas nanopartículas no se establecen protocolos para el uso de estos compuestos, por lo que se busca con ayuda de la nanometrología monitorear parámetros como el tamaño, composición química y la determinación cuantitativa de los niveles permitidos para su uso en este campo [44]. Sin embargo, otras investigaciones aportan luz con diversas técnicas que requieren validación sobre el comportamiento de las nanopartículas, como el llevado a cabo por Sousa y su equipo de trabajo, donde proponen un protocolo apoyado en TEM y SEM para el reconocimiento de la interacción entre nanopartículas y los microorganismos [45].

Wohlleben reporta la validación de la técnica de ultraprecisión analítica para determinar la capacidad de dispersión y distribución del tamaño de nanopartículas [46]. Braun et al. reportan la certificación de un material de referencia para garantizar la calidad y validez de los experimentos desarrollados a través de diferentes técnicas (Tabla 1); a su vez, los autores describen el uso de materiales de referencia no certificados que se emplean en las diferentes industrias para desarrollo de métodos y calibración, materiales de referencia aplicados en la industria y materiales de referencia para la validación parcial del método y control de la 
Tabla 1. Técnicas para la determinación del tamaño de superficie de nanopartículas y nanomateriales. Aquí se resumen las principales técnicas empleadas para medir el tamaño, la composición química, capacidad de dispersión y la distribución del tamaño de nanopartículas y nanomateriales, así como el campo en que son aplicadas, algunas ventajas y limitaciones.

\begin{tabular}{|c|c|c|c|c|}
\hline Técnica & Mediciones & Campo & Limitaciones & ventajas \\
\hline $\begin{array}{l}\text { Dispersión dinámica de } \\
\text { luz (DLS). }\end{array}$ & $\begin{array}{l}\text { Partículas en } \\
\text { solución. } \\
\text { Mezcla de } \\
\text { nanopartículas. }\end{array}$ & Toxicología. & $\begin{array}{l}\text { Rango de medida. } \\
\text { Requieren de la } \\
\text { determinación de } \\
\text { la morfología de la } \\
\text { nanopartícula. } \\
\text { Requiere trabajar con un } \\
\text { solo tamaño de partícula. }\end{array}$ & $\begin{array}{l}\text { Fáciles de usar, son } \\
\text { económicas. Tienen } \\
\text { alta sensibilidad para } \\
\text { detectar partículas en } \\
\text { solución. } \\
\text { Es una técnica no } \\
\text { invasiva. }\end{array}$ \\
\hline TEM, SEM & $\begin{array}{l}\text { Mezcla de } \\
\text { partículas de } \\
\text { diferentes } \\
\text { tamaños y } \\
\text { formas. }\end{array}$ & $\begin{array}{l}\text { Alimentos, } \\
\text { cosmética y } \\
\text { empaques } \\
\text { para alimentos, } \\
\text { toxicología. }\end{array}$ & $\begin{array}{l}\text { Costosa, presenta mayor } \\
\text { complejidad e inadecuada } \\
\text { para realizar numerosas } \\
\text { mediciones. }\end{array}$ & $\begin{array}{l}\text { Proporciona los medios } \\
\text { para la caracterización } \\
\text { de otros métodos y la } \\
\text { calibración de equipos. } \\
\text { Son muy precisas. }\end{array}$ \\
\hline $\begin{array}{l}\text { Técnicas basadas } \\
\text { en centrifugación: } \\
\text { sedimentación } \\
\text { centrífuga de partículas } \\
\text { (CPS), centrifugación } \\
\text { por sedimentación } \\
\text { líquida (CLS) y ultra } \\
\text { centrifugación analítica } \\
\text { (AUC). }\end{array}$ & $\begin{array}{l}\text { Nanopartículas } \\
\text { en suspensión. }\end{array}$ & $\begin{array}{l}\text { Industria de } \\
\text { alimentos. }\end{array}$ & $\begin{array}{l}\text { Requiere el conocimiento } \\
\text { de la densidad de la } \\
\text { nanopartícula a estudiar. } \\
\text { Presenta dificultad para } \\
\text { determinar partículas } \\
\text { inferiores a los } 20 \mathrm{~nm} \text {. }\end{array}$ & $\begin{array}{l}\text { Son más eficientes } \\
\text { en las mediciones } \\
\text { de mezclas de } \\
\text { nanopartículas con } \\
\text { diferentes tamaños. } \\
\text { Es más precisa que la } \\
\text { técnica DLS. } \\
\text { Es una técnica de bajo } \\
\text { costo. }\end{array}$ \\
\hline $\begin{array}{l}\text { Fraccionamiento de flujo } \\
\text { de campo (FFF). }\end{array}$ & $\begin{array}{l}\text { Mezcla de } \\
\text { nanopartículas. }\end{array}$ & $\begin{array}{l}\text { Medicina y } \\
\text { la industria } \\
\text { alimentaria. }\end{array}$ & $\begin{array}{l}\text { Se pueden separar y } \\
\text { medir nanopartículas de } \\
\text { diferentes tamaños. } \\
\text { Permite recuperar los } \\
\text { componentes separados. } \\
\text { No es muy precisa. } \\
\text { No distingue entre formas } \\
\text { y tamaños. } \\
\text { Las nanopartículas } \\
\text { pueden interactuar con la } \\
\text { matriz. }\end{array}$ & $\begin{array}{l}\text { Es posible acoplarla } \\
\text { a otras técnicas } \\
\text { de detección de } \\
\text { nanopartículas para } \\
\text { aumentar su presión. }\end{array}$ \\
\hline $\begin{array}{l}\text { Análisis de seguimiento } \\
\text { de nanopartículas (NTA). }\end{array}$ & $\begin{array}{l}\text { Mezcla de } \\
\text { nanopartículas. }\end{array}$ & $\begin{array}{l}\text { Industria de } \\
\text { alimentos. }\end{array}$ & $\begin{array}{l}\text { Es necesario conocer } \\
\text { la viscosidad y la } \\
\text { temperatura del medio. } \\
\text { Su precisión disminuye } \\
\text { cuando las nanopartículas } \\
\text { se encuentran en medios } \\
\text { diluidos. }\end{array}$ & $\begin{array}{l}\text { Mayor precisión en la } \\
\text { detección de partículas } \\
\text { grandes. }\end{array}$ \\
\hline
\end{tabular}

Fuente: Elaboración propia. 
calidad [47]. Se reportan diferentes técnicas para la caracterización de nanopartículas en solución por varios autores, quienes buscan determinar la toxicidad en tejidos celulares, obteniendo que la aglomeración de las nanopartículas disminuye debido a una interacción con las proteínas presenten en el cultivo celular, concluyendo que la toxicidad de la nanopartículas se debe a la composición química y a la reactividad del área superficial, así mismo reportan datos de toxicidad para diferentes nanopartículas y en distintas concentraciones [48-49]. La determinación de técnicas confiables permitirá el intercambio internacional de bienes y el desarrollo de procesos precisos basados en parámetros de trazabilidad de las medidas críticas del artículo a manufacturar, ya que al tener certificaciones bajo diferentes normas de calidad se reducen costos, se compara, se evalúa y se mejoran los procesos; siendo estas mediciones la base metrológica para el desarrollo de nuevas nanopartículas y aplicaciones, ya que de esta manera se reducen las incertidumbres asociadas a la caracterización de estos nanocompuestos [48].

Mihindukulasuriya y Lim reportan el uso de la nanotecnología en el sector industrial, especialmente en el embalaje de los alimentos y su conservación, sector donde la metrología tiene una gran aplicación dada la necesidad de realizar mediciones sobre los polímeros presentes en los empaques, de manera que no vayan a afectar la composición de los alimentos, garantizar la ausencia de agentes patógenos, los niveles de oxígeno, humedad, frescura, entre otras propiedades [50]. En este sector es de suma importancia llevar un control de los nanomateriales que son empleados, como aditivos, aromatizantes o alimentos nuevos, así como una evaluación de sus propiedades físico-químicas para garantizar que cumplen con los estándares de calidad.
Handford y colaboradores hacen referencia al uso de trituración o molienda y la homogeneización para la obtención de nanomateriales, donde la nanometrología garantizara el tamaño de partícula del nanomaterial a través de los instrumentos de medición, además describen el uso de nanosensores para el control de la calidad y el deterioro de los alimentos [51]. Dentro de las técnicas que tienen mayor aplicación en este campo se encuentran la microscopía y la espectroscopia para la determinación del tamaño de partícula y la composición química. Rossi y colaboradores mencionan que se recomienda la determinación del tamaño de partícula al menos por dos técnicas diferentes [52]. Dudkiewicz y su equipo de trabajo reportan técnicas, como SEM y TEM, para la caracterización de las nanopartículas (Figura 3); en este estudio resaltan que en la industria de alimentos no se han desarrollado investigaciones para caracterizar las nanopartículas por lo cual se toman ejemplos de la nanomedicina y la nanotoxicología [53].

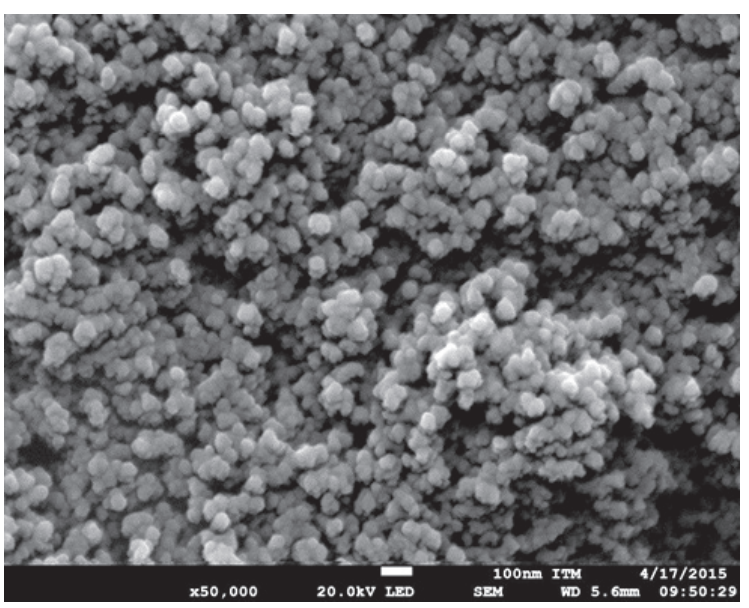

Figura 3. Nanopartículas de silice a través de SEM. Instituto Tecnológico Metropolitano (ITM), Medellín, Colombia.

Fuente: Elaboración propia. 
Linsinger y colaboradores mencionan que para que una medición pueda tener confiabilidad, debe ser posible definir la cantidad y la identidad (tamaño y composición química) de la nanopartícula presente en el alimento, reportan que la confiabilidad del resultado depende del número de partículas analizadas. Por otra parte, indican que la determinación de materiales de referencia para combinaciones de alimentos no es viable [54]. En otra investigación, llevada a cabo por Dudkiewicz et al., se propone el uso de nanopartículas de plata y sílice amorfa sintética como material de referencia para la medición de nanopartículas presentes en alimentos, así como un modelo para el cálculo de la incertidumbre y la precisión en la determinación del tamaño de partícula a través de TEM y SEM [55]. Grombe y su equipo de trabajo reportan una investigación sobre la producción de materiales de referencia para la determinación del tamaño de nanopartículas en sopa de tomate, los elementos que integran este proceso se describen en la Figura 4 [56].

\subsection{Nanometrología en Colombia}

Finalmente, se identifica que la nanometrología en Colombia es un campo que requiere de gran investigación y avances, si bien se reporta por Henao como un requisito previo para el aseguramiento de la calidad, en su investigación sobre nanociencia y nanotecnología solo hace alusión al tema en un párrafo corto [57]. Por lo que se espera que Colombia, con los grupos de investigación asociados al Centro de Excelencia de Nuevos Materiales (CENM), creado por el Gobierno Nacional y Colciencias, generen luz sobre este tema, sobre cómo es aplicado en los laboratorios académicos [58].

Si bien hay reportes que indican que Colombia invierte en investigación en nanotecnología, y los avances en este campo abordan temáticas como la nanofabricación, no se describe ningún protocolo o guía metrológica que respalde los experimentos desarrollados [59]. En Colciencias se encuentran registrados 4 grupos que trabajan en nanotecnología, en áreas como: Materiales nanoestructurados y biomodelación (MATBIOM) de la Universidad de Medellín, el cual cuenta con 5 líneas de investigación; el grupo de nanociencia y nanotecnología de la Universidad Javeriana, con 2 líneas de investigación, dentro del cual han desarrollado un estudio del uso de nanopartículas como alternativa para el suministro de fármacos, un estudio sobre nanotoxicidad, un diseño de síntesis de nanopartículas, varias investigaciones sobre el efecto de nanopartículas en el medio ambiente en: la descontaminación de aguas, sobre el efecto en las comunidades microbianas, en células epiteliales, un ensayo sobre toxicidad celular con nanotubos de carbono, varias tesis doctorales en la descontaminación de aguas con nanopartículas. Otro grupo de investigación es el de materiales avanzados para micro y nanotecnología (IMAMNT), de la Universidad Autónoma de Occidente; también se encuentra el grupo de nanomateriales multifuncionales de la Universidad de Cartagena, la cual cuenta con 5 líneas de investigación, dentro de estas hay un artículo sobre la biosíntesis y caracterización de nanopartículas, un estudio sobre el impacto ambiental en el uso de nanopartículas, varios trabajos de grado en la síntesis de nanopartículas, otros estudios en la descontaminación de aguas con nanomateriales. A pesar de que ningún grupo reporta el desarrollo de la nanometrología dentro de sus investigaciones, resulta importante destacar que para el desarrollo de estos trabajos es necesaria la caracterización metrológica y que el tema podría dar origen a investigaciones futuras. 


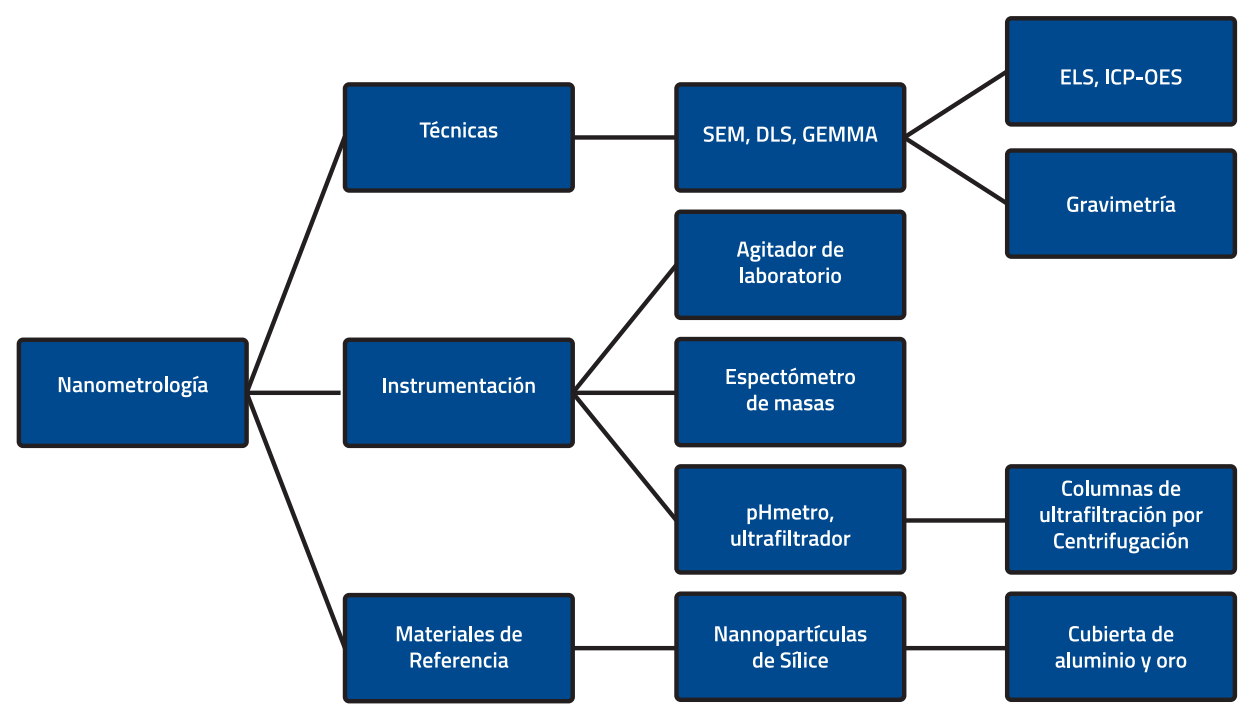

Figura 4. Elementos de un sistema de medición a nanoescala. Análisis de movilidad electroforética molecular en fase gaseosa (GEMMA). Fraccionamiento de campo de flujo junto con un espectrómetro de masas acoplado inductivamente (ICP-MS-FFF). Dispersión de luz electroforético (ELS). Espectroscopia de emisión óptica con plasma acoplado inductivamente (ICP-OES)

Fuente: Elaboración propia con base en [56].

Dentro de los grupos que están enmarcados en metrología en Colciencias se destacan: el de instrumentación y metrología de la Universidad del Atlántico, que reporta un trabajo de grado sobre la preparación y caracterización morfológica de nanopartículas, otro sobre técnicas de procesamiento y sintesis de nanomateriales. También es posible encontrar otros grupos que trabajan en otras área e integran la nanotecnología, como el de materiales y nanotecnología de la Universidad del Cauca; el grupo de superconductividad y nanotecnología de la Universidad Nacional, sede Bogotá, dentro del cual se encuentra una línea dedicada a las nanoestructuras; el grupo de nanotecnología y gestión sostenible (Nanosost-UP) de la Universidad de Pamplona, dentro del cual tiene una línea de nanociencia y nanotecnología; el grupo de ingeniería y nanotecnología para la vida (INVID), de la Universidad Distrital Francisco José de Caldas, en el cual una de sus líneas de investigación está dedicada a nanociencias; el grupo nuevos materiales nano y supramoleculares de la Universidad Nacional, sede Bogotá, tiene una línea de investigación dedicada a los nanomateriales; en este hay reportada una publicación sobre el impacto de la nanotecnología en las economías regionales, el rendimiento económico de la nanotecnología y un informe del primer acercamiento de la nanotecnología para Colombia; cuentan, además, con una publicación de la nanotecnología al servicio de la salud; finalmente, el grupo de metrología química y bioanálisis (GIMQB), del Instituto Nacional de Metrología. En general se observa que aún falta por abordar la metrología a 
escala nanométrica, desde los grupos y centros de investigación, dado que para el desarrollo de cada proyecto descrito y la funcionalidad de cada grupo es necesario todo un sistema metrológico que permita validar los experimentos y que estos puedan servir de base para el desarrollo de procesos productivos y el adelanto de proyectos que den soluciones a las necesidades industriales, sociales y reglamentarias [60].

Actualmente Ruta N desarrolla una política regional de innovación (IRI) en "nanotecnología que busca fomentar la investigación y el desarrollo de nanociencia y nanotecnología (...) para potenciar las capacidades y los recursos", así como aumentar la competitividad científica y tecnológica, con el propósito de solucionar las problemáticas de la industria, contribuir a la generación de conocimiento, integrar trabajo de diferentes sectores, impulsar la formación del recurso humano y favorecer redes de negocios. La iniciativa cuenta con " 44 instituciones, de las cuales 28 son organizaciones y 16 instituciones educativas". Dentro de esta iniciativa se desarrolló el Primer Seminario Internacional de Biomateriales, Biomecánica y Medicina Regenerativa, a través de la Universidad de Antioquia y Ruta N, lo cual genera espacios que permitan divulgar la información en desarrollo en el área de la nanomedicina, que busca crear negocios y dar a conocer los productos, con el objetivo de reunir a varios sectores y áreas de conocimiento que impulsen y generen redes de trabajo para avanzar en este campo. Otra de las actividades desarrolladas es la realización de conversatorios sobre nanotecnología y medio ambiente [61].

Dentro de las industrias que reportan investigación en el sector de nanotecnología se encuentran EPM, que apunta al desarrollo de temas como: la salud, la energía, gas natural y agua. Por otro lado está Sumicol, que busca darle características nuevas y otras propiedades a los minerales a través de nanopartículas y el desarrollo de nanomateriales para incursionar en otros mercados. En los conversatorios sobre nanotecnología se proponen el desarrollo de los siguientes sectores: "construcción, textil, farmacéutico, industrial, energía, transporte y cosmético, entre otros" [61]. En los que cada uno requiere del aporte metrológico para el desarrollo de sus actividades y el aseguramiento de la calidad, y generar la posibilidad de integrar el conocimiento y el desarrollo de proyectos académicos encaminados a solucionar las necesidades de las empresas y el fortalecimiento en el desarrollo de herramientas y equipos que permitan fabricar los productos que a su vez solucionarán las demandas de la sociedad.

Méndez y su equipo de trabajo investigan la aplicación de nanotecnología para empaques y envases para alimentos; en su investigación no se aborda la nanometrología, pero esto supone el despliegue de todo un sistema de aseguramiento metrológico tanto para el diseño de los empaques como para su fabricación, lo cual deja un campo abierto para el desarrollo de investigaciones [62].

Manrique investiga la aplicación de la nanotecnología en la industria textil a través de nanopartículas, nanotubos de carbono y nanofibras para la producción de textiles; en este campo resulta muy importante la metrología química que emplea sensores para detectar vapores al interactuar con nanopartículas, mientras que el proceso para obtener nanofibras requiere del electrohilado para producir el diámetro de estos nanohilos, transformación que requiere de una gran cantidad de herramientas que controlen el 
proceso para entregarlas bajo condiciones específicas [63].

La rednano Colombia, en su gaceta informativa, reportó el desarrollo de foros con la temática la nanotecnología para la solución a problemas de contaminación de mercurio debido a la minería artesanal, y concluyó que es necesario el fortalecimiento de la industria instrumental para la caracterización y medición. A su vez, el desarrollo de la investigación realizada por la Universidad de los Andes, la Universidad del Rosario y la Pontificia Universidad Javeriana, permitió la creación de un documento de recomendación sobre nanomateriales [64].

Por su parte, la red de laboratorios y servicios Labsnano tiene un enfoque muy básico y aún no se enfoca en la elaboración de protocolos para el uso de nanomateriales y caracterización de nanopartículas, tampoco se reportan estudios sobre los efectos del uso de estos nanomateriales sobre el medio ambiente. Actualmente la actividad desarrollada en los procesos metrológicos de los Labsnano, hacen contribución con: "actividades de capacitación y socialización de técnicas experimentales y de caracterización y manejo de equipos y procesos" [64].

Un avance en cuanto a reglamentación resulta del comité Icontec 243, creado en el 2014 para la normalización de la nanotecnología aplicada en diversos campos, el cual tendrá entre sus funciones la regulación, caracterización, medición, determinación de los impactos ambientales, y en la salud en la utilización de nanomateriales [65]. Lo cual presenta un progreso y abre la puerta a la generación de más investigaciones, aportes industriales y reglamentación para el uso de este tipo de materiales.

\section{CONCLUSIONES}

La nanometrología permite respaldar la exactitud de las mediciones realizadas a lo largo de todo el proceso de fabricación, y garantiza que el producto cumpla con los estándares de calidad, además asegura la trazabilidad de las medidas para que los componentes puedan ser integrados en el producto final, de manera que es posible certificar frente a organismos regulatorios, ya sea nacionales o internacionales, que el artículo procesado es seguro para su uso. Lo que a su vez proporciona un avance para la nanotecnología, ya que posibilita la transformación de nanodispositivos en grandes lotes, como es el área de los semiconductores, que requieren una alta precisión para ser integrados en otros componentes.

Como análisis de la literatura examinada se evidencia una dependencia directa entre el control de los procesos a nanoescala y la nanometrología, identificando así al aseguramiento de la medición como variable crítica del control de calidad. Se manifiesta que dicho control de procesos enfoca sus actividades no solo sobre la instrumentación, sino también sobre todos aquellos elementos que conforman el sistema de medida y que pueden ser fuente de incertidumbre de medición, como: procedimientos, patrones, materiales de referencia, estándares, condiciones ambientales, competencia del personal, etc.

La gran diversidad de equipos existentes para procesar nanomateriales indica la necesidad de gestionar metrológicamente los mismos y velar porque se confirmen sus tolerancias dentro del proceso productivo a través de actividades de calibración, validación, verificación, mantenimiento, entre otras, en busca de 
satisfacer las necesidades del producto durante su transformación. A su vez, se reconoce el aporte multidisciplinar que implica el desarrollo de herramientas en áreas de la ingeniería como la mecánica, los materiales, la eléctrica, el control industrial, los sistemas informáticos, la física, la química, entre otras.

Se identifica una tendencia hacia el diseño y fabricación de equipos que permitan la automatización de los procesos y su comunicación, dado que esto posibilita una disminución en los costos de fabricación y un aumento en los niveles de exactitud. Dichos sistemas automatizados a través de la metrología virtual se diseñan con el propósito de medir las propiedades de los nanomateriales a través de la caracterización y patronaje de un nanocomponente.

Queda un amplio camino por recorrer en cuanto al diseño de metodologías, validación de técnicas, certificación de materiales de referencia y caracterización de nanopartículas; con el objeto de aumentar la confiabilidad en las mediciones, principalmente en el campo médico y la industria alimentaria. Lo que posibilita el desarrollo de la metrología química y la toxicología para potenciar la aplicación de nanotubos de carbono de manera confiable.

Resulta evidente que la aplicación de técnicas y diseño de metodologías depende de la necesidad de cada sector, del nanodispositivo a fabricar y su aplicación final, por lo que aún no se cuenta con parámetros que puedan aplicarse a la manufactura de todos los nanocompuestos. Aspectos como la trazabilidad, la estimación de incertidumbre, la certificación de nanomateriales, el desarrollo de procedimientos de calibración y técnicas de validación de métodos, dependen de cada industria, la cual a través de la comunidad científica y el uso de técnicas estadísticas establece las metodologías para mejorar la confiabilidad de las mediciones, a través de la comparación de técnicas de microscopía, metrología dimensional o de espectrometría.

Actualmente la técnica que mayor aplicación tiene en todas las industrias es la microscopía, la literatura reporta un número significativo de artículos donde los autores se basan en esta técnica para la determinación de materiales de referencia y elaboración de protocolos para la caracterización de nanopartículas, principalmente en alimentos y en el campo médico, así como para observar las superficies al aplicar pruebas de nanoindentación y el grabado de caracteres a través de la nanolitografía. Mientras que otros autores prefieren técnicas de medición ópticas, por la reducción en el tiempo de ciclo del proceso y la flexibilidad que presentan.

A pesar de que se reconocen organismos de control y laboratorios que trabajan en pro de la certificación de materiales de referencia y protocolos de fabricación de nanopartículas, la nanomedicina es un campo donde aún se requiere mucha investigación, más ensayos sobre el efecto de las nanopartículas en el organismo, para el tratamiento de tumores, destrucción de células cancerígenas, su toxicidad y biodegradación, lo que invita a que se sigan realizando investigaciones que permitan la unificación de criterios en esta área. Mientras que el sector de alimentos está empezando a ganar terreno en cuanto a la superación de estas falencias.

Se observa que la mayor contribución al avance científico relacionado con nanometrología lo realizan países como: Estados Unidos, Alemania, China, Japón, Corea, Inglaterra, Brasil y México; por lo que es evidente que en 
Colombia hay ausencia de investigadores en esta área y con esto se observa una reducida importación de equipos que permiten soportar la investigación y el avance tecnológico de las regiones en el país.

\section{AGRADECIMIENTOS}

Como tutor del Semillero de Investigación en Gestión Metrológica agradezco a Estefanía Ruiz, estudiante egresada del programa Ingeniería en Producción, quien gracias a su compromiso, dedicación y buen uso de los recursos construyó un artículo con alto grado de pertinencia, el cual sirve como orientación para aquellos interesados en la medición a nanoescala y su impacto en los sistemas de producción. Se agradece a todas las personas que con sus aportes y motivación hicieron posible la realización de este trabajo, especialmente al Instituto Tecnológico Metropolitano (ITM) de Medellín por ser la entidad financiadora del trabajo.

\section{REFERENCIAS}

[1] Jorio, A. \& Dresselhaus, M. S. (2011). Nanometrology Links State of the Art Academic Research and Ultimate Industry Needs for Technological Innovation. MRS Bulletin, 32(12), pp. 988-993. doi: $10.1557 / \mathrm{mrs} 2007.201$

[2] Bogue, R. (2007). Nanometrology: a critical discipline for the twenty first century. Sensor Review, 27(3), pp. 189196. doi: $10.1108 / 02602280710758110$

[3] Ukraintsev, V. \& Banke, B. (2010). Nanoscale measurement tests metrologists. Laser Focus World, 46(12), pp. 68-71.
[4] Logothetidis, S. (2010). Nanometrology. En: Sattler, KD. Handbook of Nanophysics. Nueva York, Estados Unidos: CRC Press, pp. 29-45.

[5] Ukraintsev, V.\&Banke, B. (2012). Review of reference metrology for nanotechnology: significance, challenges, and solutions. Journal of Micro/Nanolithography, MEMS, and MOEMS, 11(1), pp. 11010-11019. doi: 10.1117/1.jmm.11.1.011010

[6] Berthold, J. \& Imkamp, D. (2013). Looking at the future of manufacturing metrology: roadmap document of the German VDI/ VDE Society for Measurement and Automatic Control. J. Sensors Sens. Syst., 2, pp. 1-7. doi: 10.5194/jsss-2-1-2013

[7] Jorio, A. \& Dresselhaus, M. S. (2008). Nanometrology Sees Progress in Synthesis, Optics, and Microscopy. MRS Bulletin, 33(10), p. 972. doi: 10.1557/ mrs2008.208

[8] Garner, C. M. \& Vogel, E. M. (2006). Metrology Challenges for Emerging Research Devices and Materials. IEEE Transactions on Semiconductor Manufacturing, 19(4), pp. 397-403. doi: 10.1109/tsm.2006.884714

[9] Yushchenko, O. V. \& Yurko, D. S. (2015). Investigation of Plastic Deformation Considering Nanoscale Effects. Nanomaterials: Application \& Properties, 4(1), pp. 1-3.

[10] Barker, K. E., Cox, D. \& Sveinsdottir, T. (2011). Foresight on the future of public research metrology in Europe. Foresight, 13(1), pp. 5-18. doi: 10.1108/14636681111109660 
[11] Barsic, G., Simunovic, V. \& Katic, M. (2011). Ensuring measurement unity in the field of dimensional nanometrology. Annals of DAAAM \& Proceedings, 22 (1), pp. 841-842.

[12] Carreteiro-Damasceno, J., Ribeiro, A. R., Balottin, L. B. L. \& Granjeiro, J. M. (2013). Nanometrology - challenges for health regulation. Vigilância Sanitária em Debate Soc. Ciência \& amp; Tecnol., 1(4), pp. 100109. doi: 10.3395/vd.v1i4.94en

[13] Gavrilenko, V. P., Novikov, Y. A., Rakov, A. V. \& Todua, P. A. (2008). Metrology and Standardization For Nanotechnologies. En AIP Conference Proceedings, 99 (1), pp. 286-297. doi: 10.1063/1.2918114

[14] Kumar, A. \& Jee, M. (2013). Nanotechnology: A Review of Applications and Issues. International Journal of Innovative Technology and Exploring Engineering (IJITEE), 3(4), pp. 1-2.

[15] Chang, C., Bukkapatnam, S. \& Komanduri, R. (2014). Sensing and Informatics in Laser-Based Nanomanufacturing Processes. En: Nof S.Y., Weiner A.M. \& Cheng G.J. Laser and Photonic Systems: Design and Integration. New York. CRC Press. pp. 201-234.

[16] Gheorghe, G. I. \& Badita, L.-L. (2013). Micro-Nanometrologically and Topographic Characterization of Nanostructured Surfaces. Procedia Engineering, 69, pp. 104-111. doi: 10.1016/j.proeng.2014.02.209

[17] Salamon, A. W. (2011). Characterizing Engineered Nanomaterials. R\&D Mag, 53 (2), pp. 24.
[18] Weckenmann, A., Krämer, P. \& Akkasoglu, G. (2012). Metrology base for scientific cognition and technical production. En AIP Conf. Proc., 1431, pp. 283-292. doi: 10.1063/1.4707576

[19] Kang, P., Kim, D., Lee, H., Doh, S. \& Cho, S. (2011). Virtual metrology for run-to-run control in semiconductor manufacturing. Expert Syst. Appl., 38(3), pp. 2508-2522. doi: 10.1016/j.eswa.2010.08.040

[20] Diebold, A. C. (2005). Metrology (including Materials Characterization) for Nanoelectronics. En AIP Conf. Proc, 788(1), pp. 21-32. doi: 10.1063/1.2062935

[21] Töpfer, S. C. N., Nehse, U. \& Linß, G. (2007). Automated inspections for dimensional micro- and nanometrology. Measurement, 40(2), pp. 243-254. doi: 10.1016/j.measurement.2006.06.010

[22] Kang, N., Kim, K. J., Kim, J. S. \& Lee, J. H. (2015). Roles of chemical metrology in electronics industry and associated environment in Korea: A tutorial. Talanta, 134, pp. 284-91. doi: 10.1016/j.talanta.2014.11.030

[23] Pimpin, A. \& Srituravanich, W. (2012). Review on Micro- and Nanolithography Techniques and Their Applications. Engineering Journal, 16(1), pp. 37-65. doi: 10.4186/ej.2012.16.1.37

[24] Kang, P., Lee, H., Cho, S., Kim, D., Park, J., Park, C.-K., \& Doh, S. (2009). A virtual metrology system for semiconductor manufacturing. Expert Syst. Appl., 36(10), pp. 12554-12561. doi: 10.1016/j.eswa.2009.05.053

[25] Liu, S., Chen, X. \& Zhang, C. (2015). Development of a broadband Mueller 
matrix ellipsometer as a powerful tool for nanostructure metrology. Thin Solid Films, 584, pp. 176-185. doi: 10.1016/j. tsf.2015.02.006

[26] Likhachev, D. V. (2015). Efficient thin-film stack characterization using parametric sensitivity analysis for spectroscopic ellipsometry in semiconductor device fabrication. Thin Solid Films, 589, pp. 258263. doi: 10.1016/j.tsf.2015.05.049

[27] Khan, A. A., Moyne, J. R. \& Tilbury, D. M. (2008). Virtual metrology and feedback control for semiconductor manufacturing processes using recursive partial least squares. J. Process Control, 18(10), pp. 961-974. doi: 10.1016/j. jprocont.2008.04.014

[28] Susto, G. A., Pampuri, S., Schirru, A., Beghi, A. \& De Nicolao, G. (2015). Multi step virtual metrology for semiconductor manufacturing: A multilevel and regularization methodsbased approach. Computers \& Operations Research, 53, pp. 328-337. doi: 10.1016/j.cor.2014.05.008

[29] Slocum Jr., A. H. \& Culpepper, M. L. (2012). Design of a low-cost, precision belt-drive machine for high-throughput nanomanufacturing. Precision Engineering, 36(1), pp. 55-69. doi: 10.1016/j. precisioneng.2011.07.003

[30] Malshe, A. P., Rajurkar, K. P., Virwani, K. R., Taylor, C. R., Bourell, D. L., Levy, G., Sundaram, M. M., McGeough, J. A., Kalyanasundaram, V. \& Samant, A. N. (2010). Tip-based nanomanufacturing by electrical, chemical, mechanical and thermal processes. CIRP Ann. -
Manuf. Technol, 59(2), pp. 628-651. doi: 10.1016/j.cirp.2010.05.006

[31] Hernández-Santana, A. \& Graham, D. (2010). Nanolithography: Written with light. Nat. Nanotechnol, 5(9), pp. 629-630. doi: 10.1038/nnano.2010.179

[32] Campbell, A. C., Klapetek, P., Valtr, M. \& Buršíková, V. (2012). Development of reference materials for the investigation of local mechanical properties at the nanoscale. Surf. Interface Anal, 44(8), pp. 1151-1154. doi: 10.1002/sia.4850

[33] Lucca, D. A., Herrmann, and K. \& Klopfstein, M. J. (2010). Nanoindentation: Measuring methods and applications. CIRP Ann. - Manuf. Technol, 59(2), pp. 803819. doi: 10.1016/j.cirp.2010.05.009

[34] Xia, Y., Bigerelle, M., Marteau, J., Mazeran, P. E., Bouvier, S. \& Lost, A. (2013). Effect of surface roughness in the determination of the mechanical properties of material using nanoindentation test. Scanning, 36(1), pp. 134-149. doi: 10.1002/sca.21111

[35] Li, Z., Herrmann, K. \& Pohlenz, F. (2006). A comparative approach for calibration of the depth measuring system in a nanoindentation instrument. Measurement, 39(6), pp. 547-552. doi: 10.1016/j.measurement.2005.12.010

[36] Qiu, W., Li, S.-L., Deng, W.-L., Gao, D. \& Kang, Y.-L. (2014). Strain sensor of carbon nanotubes in microscale: from model to metrology. The Scientific World Journal, 2014, pp. 1-9. doi: 10.1155/2014/406154

[37] Dai, L., Wang, P. \& Bosnicka, K. (2009). Large scale production and metrology of 
vertically aligned carbon nanotube films. Journal of Vacuum Science \& Technology A: Vacuum, Surfaces, and Films, 27(4), pp.1071-1075. doi: 10.1116/1.3148827

[38] Wood, J. (2004). Nanotubes light up the home. Materials Today, 7(9), p. 11. doi: 10.1016/s1369-7021(04)00383-9

[39] Upadhyayula, V. K. K., Ghoshroy, S., Nair, V. S., Smith, G. B., Mitchell, M. C. \& Deng, S., (2008). Single Walled Carbon Nanotubes as Fluorescence Biosensors for Pathogen Recognition in Water Systems. Research Letters in Nanotechnology, 2008, pp. 1-5. doi: 10.1155/2008/156358

[40] Li, L., Hong, M., Schmidt, M., Zhong, M., Malshe, A., Huis in'tVeld, B. \& Kovalenko, V. (2011). Laser nano manufacturing State of the art and challenges. CIRP Ann. - Manuf. Technol, 60(2), pp. 735-755. doi: 10.1016/j.cirp.2011.05.005

[41] Demircioglu, P. (2014). Estimation of surface topography for dental implants using advanced metrological technology and digital image processing techniques. Measurement, 48, pp. 43-53. doi: 10.1016/j.measurement.2013.10.036

[42] Coelho, P. G., Jimbo, R., Tovar, N. \& Bonfante, E. A. (2015). Osseointegration: Hierarchical designing encompassing the macrometer, micrometer, and nanometer length scales. Dental Materials, 31(1), pp. 37-52. doi: 10.1016/j.dental.2014.10.007

[43] Kaur, A., Kaur, M. A. \& Shahi, M. N. (2012). How nanotechnology works in medicine. International Journal of Electronics and Computer Science Engineering, 1(4), pp. 2452-2459.
[44] Chakarvarty, G. (2013). Nanoparticles \& Nanotechnology: Clinical, Toxicological, Social, Regulatory \& other aspects of Nanotechnology. Journal of Drug Delivery and Therapeutics, 3(4), pp. 138-141.

[45] Sousa, C., Sequeira, D., Kolen'ko, Y. V, Pinto, I. M. \& Petrovykh, D. Y. (2015). Analytical Protocols for Separation and Electron Microscopy of Nanoparticles Interacting with Bacterial Cells. Analytical Chemistry, 87(9), pp. 4641-4648. doi: 10.1021/ac503835a

[46] Wohlleben, W. (2012). Validity range of centrifuges for the regulation of nanomaterials: from classification to as-tested coronas. J. Nanoparticle Res, 14(12), pp. 1-18. doi: 10.1007/s11051012-1300-z

[47] Braun, A., Kestens, V., Franks, K., Roebben, G., Lamberty, A. \& Linsinger, T. (2012). A new certified reference material for size analysis of nanoparticles. J. Nanoparticle Res, 14(9), pp. 1-12. doi: 10.1007/ s11051-012-1021-3

[48] Calzolai, L., Gilliland, D. \& Rossi, F. (2012). Measuring nanoparticles size distribution in food and consumer products: a review. Food Additives \& Contaminants: Part A, 29(8), pp. 1183-1193. doi: $10.1080 / 19440049.2012 .689777$

[49] Murdock, R. C., Braydich-Stolle, L., Schrand, A. M., Schlager, J. J. \& Hussain, S. M. (2008). Characterization of nanomaterial dispersion in solution prior to in vitro exposure using dynamic light scattering technique. Toxicological Sciences, 101(2), pp. 239-253. doi: 10.1093/toxsci/kfm240 
[50] Mihindukulasuriya, S. D. F. \& Lim, L.-T. (2014). Nanotechnology development in food packaging: A review. Trends Food Sci. Technol, 40(2), pp. 149-167. doi: 10.1016/j.tifs.2014.09.009

[51] Handford, C. E., Dean, M., Henchion, M., Spence, M., Elliott, C. T. \& Campbell, K. (2014). Implications of nanotechnology for the agri-food industry: Opportunities, benefits and risks. Trends Food Sci. Technol, 40(2), pp. 226-241. doi: 10.1016/j. tifs.2014.09.007

[52] Rossi, M., Cubadda, F., Dini, L., Terranova, M. L., Aureli, F., Sorbo, A. \& Passeri, D. (2014). Scientific basis of nanotechnology, implications for the food sector and future trends. Trends Food Sci. Technol, 40(2), pp. 127-148. doi: 10.1016/j.tifs.2014.09.004

[53] Dudkiewicz, A., Tiede, K., Loeschner, K., Jensen, L. H. S., Jensen, E., Wierzbicki, R., Boxall, A. B. A. \& Molhave, K. (2011). Characterization of nanomaterials in food by electron microscopy. Trac Trends Anal. Chem, 30(1), pp. 28-43. doi: 10.1016/j. trac.2010.10.007

[54] Linsinger, T. P. J., Chaudhry, Q., Dehalu, V., Delahaut, P., Dudkiewicz, A., Grombe, R., von der Kammer, F., Larsen, E. H., Legros, S., Loeschner, K., Peters, R., Ramsch, R., Roebben, G., Tiede, K. \& Weigel, S. (2013). Validation of methods for the detection and quantification of engineered nanoparticles in food. Food Chemistry, 138(2-3), pp. 195966. doi: 10.1016/j.foodchem.2012.11.074

[55] Dudkiewicz, A., Boxall, A. B. A., Chaudhry, Q., Mølhave, K., Tiede, K., Hofmann, P. \& Linsinger, T. P. J. (2015).
Uncertainties of size measurements in electron microscopy characterization of nanomaterials in foods. Food Chemistry, 176, pp. 472-479. doi: 10.1016/j. foodchem.2014.12.071

[56] Grombe, R., Charoud Got, J., Emteborg, H., Linsinger, T. P. J., Seghers, J., Wagner, S., von der Kammer, F., Hofmann, T., Dudkiewicz, A., Llinás, M., Solans, C., Lehner, A. \& Allmaier, G. (2014). Production of reference materials for the detection and size determination of silica nanoparticles in tomato soup. Anal. Bioanal. Chem, 406(16), pp. 3895-3907. doi: 10.1007/s00216-013-7554-1

[57] Henao-Duque, S. M. (2010). Nanotecnología. J. Cienc. e Ing., 2(2), pp. 7-14.

[58] Zuluaga-Vidal, D. (2007). Informe de vigilancia tecnológica: métodos de fabricación de nanotecnología. Bogotá. Colciencias, pp. 33-92.

[59] Pontificia Universidad Javeriana. (2016). Nanociencia y Nanotecnología. En: http://ingenieria.javeriana.edu.co/ investigacion/grupos-investigacion/ naciencia-natecno (26 febrero de 2016).

[60] Colciencias. Plataforma ScienTI-Colombia Colciencias. (2016). En: http://www. colciencias.gov.co/scienti (11 mayo del 2016).

[61] Ruta N. Ruta N nanotecnología. En: http:// rutanmedellin.org/es/noticias/tag/ nanotecnolog\%C3\%ADa (11 de mayo de 2016). 
[62] Méndez-Naranjo, K. C., Caicedo Palacios, M. L., Bedoya Correa, S. M., Ríos Mesa, A., Zuluaga Gallego, R. \& Giraldo Ramírez, D. P. (2014). Tendencias investigativas de la nanotecnología en empaques y envases para alimentos. Rev. Lasallista Investig, 11(2), pp. 18-28.

[63] Manrique, H. (2009). Aplicación de nanotecnología en la industria textil colombiana. Revista Virtual Pro, 11(2), pp. 18-28.

[64] Rednano Colombia. (2014). Gaceta Informativa, Vol 1. En: http:// rednanocolombia.org/gaceta04.htm (26 febrero de 2016).

[65] Rednano Colombia. (2015). Gaceta Informativa, Vol 2. En: http:// rednanocolombia.org/gaceta1 03.htm (26 febrero de 2016). 